\title{
Clinical investigation of the interface pressure in the trans-tibial socket with Dermo and Seal-In X5 liner during walking and their effect on patient satisfaction
}

\author{
Sadeeq Ali , Noor Azuan Abu Osman, Niyousha Mortaza, Arezoo Eshraghi, \\ Hossein Gholizadeh, Wan Abu Bakar Bin Wan Abas \\ Department of Biomedical Engineering, Faculty of Engineering, University of Malaya, Malaysia \\ Introduction
}

Transtibial amputation patients need prosthetic devices after amputation surgery in order to regain their functional mobility and appearance (Wolf et al., 2009). The socket design plays a significant role in determining the quality of the fit and provides an interface between the prosthesis and the residual limb (Jia et al., 2004). Appropriate socket fitting in prosthetic devices can have a significant effect on the patient's comfort, mobility and level of satisfaction with their prosthesis (Kristinsson, 1993; McCurdie et al., 1997).

Skin problems are common in prosthetic users and these can appear in the formof rashes, ulcers, irritation and allergies. Their presence is commonly attributed to one of several reasons: the inadaptability of the skin, due to the intolerance of pressure by the prosthetic socket on the residual limb; bacterial proliferation as a result of a snugly-fitted socket that causes entrapment of perspiration in a closed environment; skin irritation or allergic reaction due to the materials used in the prosthetic socket and liners (Dudek et al., 2005; Dudek et al., 2006). Lower limb amputees commonly experienced residual limb skin problems with the use of the prostheses (Laing et al., 2011). Amputees often need to stop using the prosthesis entirely for a period of time as a result of the pain and discomfort caused by such skin problems. This condition can badly effect thementalwellbeing of a patient and will ultimately impact their satisfaction with a device (Meulenbelt et al., 2006).

It is crucial that the risk of these skin complications is taken into consideration during the design of the prosthetic socket and that the design of the device is based on a good understanding of the pressure that can occur between the amputee's residual limb and the prosthetic socket (Jia et al., 2008). In order to reduce the possibility of these skin issues occurring, liners are fit inside the socket to provide the residual limbwith a soft cushion. Liners have a direct contact with the residual limb inside the socket and play a significant role in transferring the load and distributing 
the interface pressure over the residual limb (Coleman et al.,

2004; Lin et al., 2004).

Polyethylene foam linerswith patellar tendon bearing (PTB) prosthetic

socket have been in use since 1950; however, modern liners, which are

generally made from silicone and other elastomers, offer better suspension

and cushion (Dietzen et al., 1991; Haberman et al., 1992; Madigan

and Fillauer, 1991). Silicon and gel liners were introduced worldwide in

themid 1990s andwere designed to reduce shear forces and produce better

interface bonds between the residual limb and the socket (Van deWeg and Van Der Windt, 2005). One of these silicone liners is known as the

Seal-In X5 liner (Fig. 1). It was introduced by Ossur (Reykjavik, Iceland)

and is composed of five seals that conform to the shape of the internal

socket wall and the residual limb (Gholizadeh et al., in press). Through

this, the Seal-In X5 liner provides suspension without the need for an external

sleeve or lock and claim to be a good choice for high impact activities.

The Dermo liner (Reykjavik, Iceland) is also made of silicone;

however, unlike the Seal-In X5 liner, it cushions the limb and provides

suspension through a shuttle lock system (Fig. 1).

Many studies have been carried out to investigate the interface pressure

and stresses (Jia et al., 2005; Sanders et al., 1998; Wolf et al., 2009).

Some of them compared the socket pressure of polyethylene foam liners

with silicone liners (Dumbleton et al., 2009). Some studies have investigated

the effect of various casting techniques or socket design on the

socket-residual limb interface pressure (Dumbleton et al., 2009; Jia

et al., 2005; Lee and Zhang, 2007), while other studies have focused on

the effect of alignment on interface pressure (Jia et al., 2008). However,

none of these studies compared the effect of a Dermo liner that used a

shuttle lock with a sealing system such as the Seal-In X5 liner. In the

Seal-In X5 liner, the seals have the potential to impose extra pressure

over the residual limb. This can cause excessive pressure, that in it can

be a source of problems for diabetic patients or amputees with sensitive residual limbs. The aim of this clinical studywas to measure and evaluate

the interface pressure in the Dermo liner during normal walking and

compare it with the Seal-In X5 liner. The study also aimed to assess the

effect that the two liners had on patients' satisfaction.

\section{Methodology}

\subsection{Subjects}

A total of nine unilateral transtibial amputees ( 7 males, 2 females)

participated in this study. All the subjects were selected fromthe Department

of Rehabilitation of the University Malaya Medical Centre (UMMC),

Kuala Lumpur, Malaysia. The ethics committee of UMMC approved this

study, and informed written approval was attained fromall the subjects.

The inclusion criteria consisted of a minimum $15 \mathrm{~cm}$ residual limb length

(from the mid patella to the distal end of residual limb), no wound and 
ulcers in the residual limb, no volume changes, and the ability to walk without the use of assistive devices. Itwas a requirement that the participants are experienced prosthetic users (more than 6 months). A sample of convenience is used for this study.

\subsection{Prosthetic interventions}

Two transtibial prostheses were made for each subject, one with the Dermo liner with shuttle lock (Icelock-200 series) and another with the Seal-In X5 linerwith valve (Icelock Expulsion, Valve 551). All the prostheses were fabricated with Flex-Foot Talux (Ossur, Reykjavik, Iceland). One registered prosthetist fabricated all the prostheses to avoid alterations due to manufacturing, alignment and fitting. A total surface bearing (TSB) socket was fabricated for all the subjects (Staats and Lundt, 1987). In order to become familiar with their new prosthetic devices, the subjects practiced walking in the motion analysis laboratory (Biomedical

Engineering Department, University of Malaya, Malaysia) and the prosthetist adjusted the fitting of the socket and alignment according to their needs. Subjects were required to use their prostheses for a minimum of four weeks. The subjects were asked to visit the brace and limb laboratory for followup on a weekly basis to ensure that the fit of theprosthesis remained suitable.

2.3. Experimental setting and procedures

After fourweeks of acclimation, the subjects attended the motion laboratory for pressure measurements. Four F-Socket sensors arrays 9811

(Tekscan Inc., South Boston, USA) were attached to the residual limb. The sensor arrays were positioned on the anterior, posterior, medial and lateral aspects of the residual limb (Fig. 1). The mid patella was taken as the reference line for the placement of medial, lateral and anterior sensors. The posterior sensor was positioned approximately $1 \mathrm{~cm}$ above the posterior trimline of the socket. Each sensor was trimmed to fit to the residual limb contours. To prevent sensor arrays displacement, the residual limb was covered with a cellophane cover. Following this, each sensor was attached to the cellophane covers by an adhesive spray (3M Spray Mount Adhesive, $3 \mathrm{M}$ corporate, St. Paul, USA). This sensor arrangement provided a pressure map that covered $90 \%$ of the residual limb during the gait. Tekscan software version 6.51 was used to record the interface pressure.

A Tekscan pressure bladder (PB100T, South Boston, USA)was used to equilibrate and calibrate the sensor arrays. Sensor arrays were placed inside the bladder and, according to the manufacturer's instructions, were subjected to a pressure of $100 \mathrm{kPa}$. Calibration was carried out based on each subject's body weight. That is, the applied pressure for calibration was the ratio of the subject's body weight to the respective sensor area (Buis, 1997). 
2.4. Walkway and collection of the data

Subjects were asked to walk at a self-selected speed on a walkway thatwas 9-meter long and 5-meter wide. Prior to the data collection activity, the subjects were requested to walk on the walkway to familiarize with the procedure. Data acquisition was performed for 12 seconds with a sample rate of $50 \mathrm{~Hz}$. The subjects completed four consecutive trials on the walkway and in each trial approximately eight to nine steps were taken. The middle step of each trial was chosen. The mean peak pressures (MPP) of four trials were employed for the purposes of statistical analyses.

\subsection{Questionnaire}

After the experiments were completed, each subject completed a questionnaire that asked for further information about their satisfaction with the two liners. Various parts of the Prosthetics Evaluation Questionnaire (PEQ) were adopted for this questionnaire. The questionnaire was composed of the following three sections:

1- Demographic variables (sex, age, weight, height, amputation side, cause of amputation, activity level and time since first prosthesis).

2- Satisfaction (fitting, donning and doffing, suspension, sitting, walking on level surfaces, ascending and descending stairs, walking on uneven ground, cosmesis and overall satisfaction).

3- Problems (Wound, skin irritation, sweating, pistoning, rotation, residual limb swelling, smell, sounds and residual limb pain).

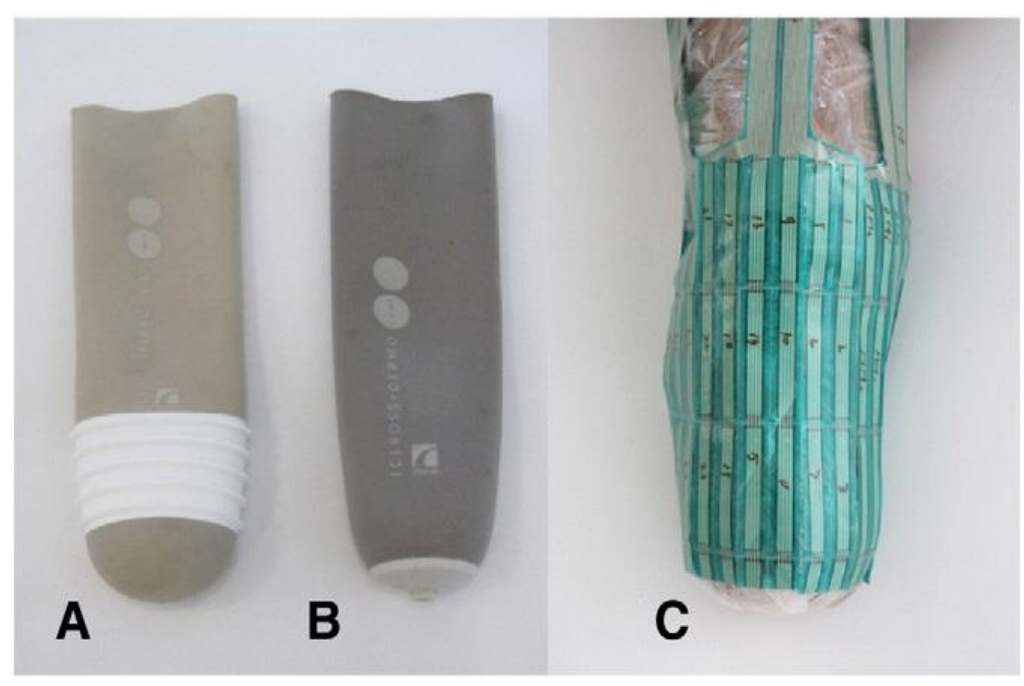

Fig. 1. (A) Seal-In Liner (B) Dermo Liner (C) Sensors attachments on residual limb.

A scale of 0-100 was used to score all the questions, where 100 indicated "complete satisfaction or no problems" and 0 indicated "unsatisfied 
or extremely bothered."

2.6. Analysis of data

Since the sample size of this studywas small $(\mathrm{N}=9)$, non-parametric

test were used to analyze the data. Therefore we used Wilcoxon signed

ranks test to compare within-subject pressure measurements with the

Dermo liner and Seal-In X5 liner for different regions in the socket. We

also used Wilcoxon signed rank test to compare the satisfaction with

the two liners. For the overall scores, which were distributed normally,

paired-samples t-test was applied. Statistical analyses were carried out

using Version 20 of SPSS, statistical software (SPSS, Chicago, IL).

\section{Results}

3.1. Subject's Profile

Themean age of the subjectswas (mean $=49.3, \mathrm{SD}=15.0$ ) and their activity level, based on the Medicare Functional Classification Level (MFCL) (Dudek et al., 2008), was K2-K3 and K3-K4. All the subjects had undergone amputation surgery at least three and half years prior to the study. The participants' demographic information is shown in Table 1.

3.2. Interface pressure

Pressure measurements were extracted in twelve regions of the residual limb. The mean of peak pressures are presented separately in

Table 2. The pressures of the four major regions of the residual limb are presented in Fig. 2. In both the anterior and posterior regions, the mean pressures for the proximal, middle subregion areas were significantly higher (Pb0.05) with the Seal-In X5 liner than they were with the Dermo liner. In both the lateral and medial regions, the pressure in the middle and distal subregion area was significantly higher (Pb0.05).

The MPP for the four major regions of the residual limb was also obtained. The MPP values for the whole anterior region of the residual limb was significantly higher for the Seal-In X5 liner compared to the Dermo liner $(\mathrm{P}=0.008, \mathrm{Z}=-2.66$; mean $=84.90 \mathrm{kPa}, \mathrm{SD}=30.46$; mean $=60.2 \mathrm{kPa}, \mathrm{SD}=13.00$, respectively). Moreover, at the posterior region, MPP was significantly higher with the Seal-In X5 liner compared to the Dermo liner $(\mathrm{P}=0.046, \mathrm{Z}=-1.99$; mean $=74.51 \mathrm{kPa}$, $\mathrm{SD}=12.04$; mean=54.10 kPa, $\mathrm{SD}=11.21$, respectively). There was a statistically significant difference between the pressure values for the two liners in the medial region of the residual $\operatorname{limb},(\mathrm{P}=0.025, \mathrm{Z}=$ -2.24; Dermo: mean=50.00 kPa, $\mathrm{SD}=12.34$; Seal-In X5: mean= $53.80 \mathrm{kPa}, \mathrm{SD}=9.45)$. There was no statistically significant difference between the pressure values for the two liners in the lateral regions of the residual limb $(\mathrm{P}=0.601, \mathrm{Z}=-0.42$; Dermo: mean $=50.00 \mathrm{kPa}$,

$\mathrm{SD}=11.21$; Seal-In X5: mean=51.50 kPa, SD=7.70) (Fig. 3). 
3.3. Questionnaire

In five out of the nine questions on the satisfaction scale of the questionnaire,

theWilcoxon Signed Rank Test revealed statistically significant

higher scores for the Dermo liner than those for the Seal-In X5 liner.

However, the Seal-In X5 liner scored better on the question about the

suspension of the prosthesis (Table 3).

In the element of the questionnaire that was aimed at assessing

problems with a device, theWilcoxon Signed Rank test showed significantly

higher scores across five items for the Dermo liner and two items

(including pistoning within the socket and unwanted sounds) for the

Seal-In X5 liner (Table 3).

The overall scores (average) of the two scales of the questionnaire

were also calculated and compared for the two liners. A paired-samples

t-test was performed to compare the scores of satisfaction and problems

scales for the Dermo and Seal-In liners. In both scales, the subjects

assigned significantly higher scores to the Dermo liner (Pb0.05) than

they did to the Seal-In liner.

Full text is available at :

http://ac.els-cdn.com/S0268003312001295/1-s2.0-S0268003312001295-main.pdf? tid=b535ef6a-a017-11e3-

954c-00000aab0f6b\&acdnat $=1393551123$ b190186c0466705bdfb3ca10943a04f1

http://www.ncbi.nlm.nih.gov/pubmed/22795863

http://www.sciencedirect.com/science/article/pii/S0268003312001295 